\title{
Salivary gland enlargement during oesophageal stricture dilatation
}

\author{
DERRICK MARTIN \\ M.B., M.R.C.P.
}

\author{
Department of Medicine, Withington Hospital, Manchester
}

\begin{abstract}
Summary
A case of recurrent salivary gland enlargement occurring during fibreoptic oesophagoscopy and oesophageal stricture dilatation with Eder-Puestow dilators is described. The genesis of this condition is discussed and its transient and usually benign nature emphasized.
\end{abstract}

\section{Introduction}

Transient, painless salivary gland enlargement has been reported as occurring during anaesthesia (Attas, Sabawala and Keats, 1968), bronchoscopy (Blackford, 1944) and rigid (Slaughter and Boyce, 1969) and flexible (Shields et al., 1977) upper gastrointestinal endoscopy. Its occurrence during oesophageal stricture dilatation using Eder-Puestow oesophageal dilators is now described.

\section{Case report}

A 50-year-old, white, male storekeeper presented with a 3-month history of epigastric pain relieved by antacids with associated dysphagia for solid foods. He had been an insulin-dependent diabetic for 10 years, taking enzyme supplements for painless, chronic pancreatitis. There was also a history of ischaemic heart disease and intermittent claudication. He was on no other medication.

Clinical examination showed a thin man with no lymphadenopathy. The cardiovascular system was normal apart from the absence of foot pulses. Abdominal examination was normal and he had no evidence of peripheral or autonomic neuropathy. Barium swallow showed an irregular stricture of the lower third of the oesophagus with some proximal dilatation. Following premedication with atropine $0.5 \mathrm{mg}$ i.m., pharyngeal lignocaine spray and diazepam $7 \mathrm{mg}$ i.v., endoscopy was performed using the Fuginon FGQBF end-viewing fibrescope. A smooth tapering, impassable stricture at $37 \mathrm{~cm}$ was found. Brush cytology and biopsy specimens showed no evidence of malignancy. The patient suffered no ill effects from this oesophagoscopy.

One week later dilatation of the stricture was undertaken using Eder-Puestow dilators with the Fuginon end-viewing fibrescope. Premedication was as before except that $12.5 \mathrm{mg}$ of diazepam were given i.v. No problems were encountered during the dilatation and the patient did not cough or retch unduly. Immediately following the procedure considerable bilateral swelling of parotid and submandibular salivary glands was noted (Fig. 1). This was painless, non-crepitant and there was no excess salivation. Serum amylase was normal. The swelling subsided spontaneously within $12 \mathrm{hr}$. Two weeks later the dilatation was repeated because of recurrent dysphagia. The procedure was as before and identical salivary gland enlargement was noted.

After one month, rigid oesophagoscopy was performed because of recurrent dysphagia. Biopsies at this time showed poorly differentiated adenocarcinoma. Three weeks later he developed mediastinitis with septicaemia and died. Post-mortem showed adenocarcinoma of the stomach involving the lower end of the oesophagus with a metastasis in the left cerebral hemisphere. There was severe mediastinitis. The salivary glands were not examined.

\section{Discussion}

Various theories have been proposed to explain this condition. Reilly (1970) described 3 patients with parotid gland enlargement following general anaesthesia. He considered the most likely predisposing factors to be the use of anticholinergic drugs, neuromuscular blocking agents and straining and coughing. Matsuki, Wakayama and Oyama (1975) also considered coughing and straining of importance. However, no individual drug or group of drugs has been commonly used in patients described in other reports and challenge with some of these drugs has not reproduced the condition (Blackford, 1944; Shields et al., 1977). Coughing and straining is an inconstant feature in other reported cases and did not occur unduly in the present case.

Slaughter and Boyce (1969) described 2 patients who developed submandibular gland enlargement after fibreoptic endoscopy and felt that this was due to mechanical occlusion of the duct orifices during the procedure. However, Slaughter (1975) later described a patient with unilateral parotid gland swelling after endoscopy and found it difficult 


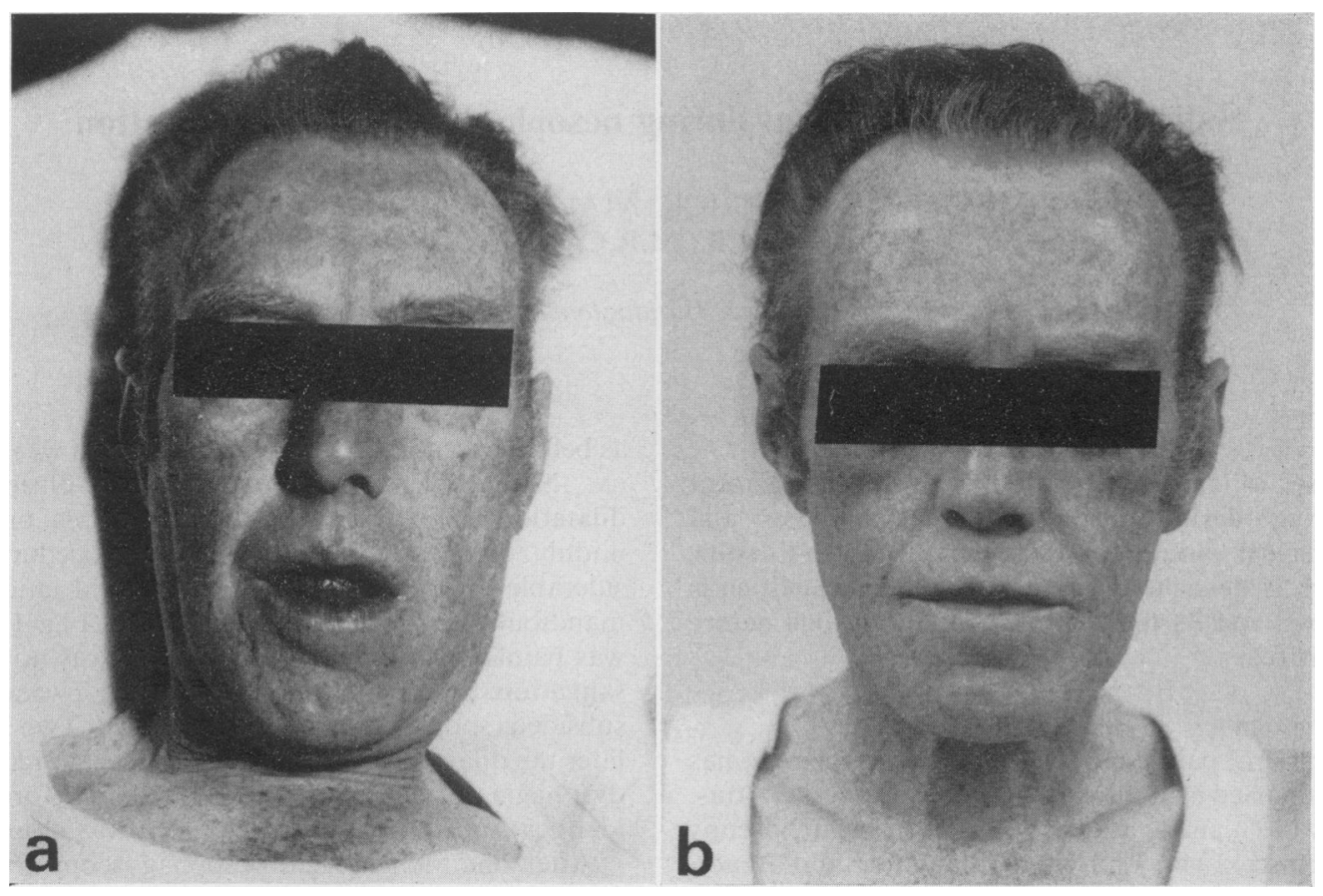

FIG. 1. Photograph of patient taken (a) immediately and (b) $24 \mathrm{hr}$ after oesophageal stricture dilatation.

anatomically to ascribe this to duct occlusion. It seems similarly difficult to explain recurrent enlargement of both parotid and submandibular glands in the present patient on the basis of mechanical obstruction of ducts.

Blackford (1944) and Bonchek (1969) felt that a reflex arc with afferent stimuli arising in the mouth or pharynx and an abnormally intense efferent response via the facial or glossopharyngeal nerves leading to vasodilatation and swelling of salivary glands was responsible for this phenomenon. Blackford supported this by showing that subcutaneous methacholine, a powerful parasympathomimetic, could induce intense salivation and swelling of the parotid and submandibular glands. However, if the afferent stimulus arises mainly from the mouth or pharynx then this condition would be expected occasionally following dental treatment but this has not been reported.

All but 2 of the reported cases (Reilly, 1970; Attas et al., 1968) have had instrumentation of the oesophagus or trachea. Possibly the autonomic reflex has its stimulus arising mainly below the level of the epiglottis explaining the rarity of the condition in dentistry and anaesthesia maintained by mask and pharyngeal airway.

If this explanation is correct then the condition may be seen more frequently by endoscopists using Eder-Puestow dilators.

There is no common underlying pathology in the patients described, neither is there any racial preponderance, although the condition appears congmoner in men.

Unexpected swelling of the face or neck following oesophageal stricture dilatation is worrying for endoscopist and patient. However, if the swelling non-crepitant and due to salivary gland enlargement then those concerned can be reassured that it wil subside spontaneously.

\section{Acknowledgment}

I thank Dr C. G. Beardwell for permission to report deta of this case.

\section{References}

Attas, M., Sabawala, P.B. \& Keats, A.S. (1968) Acute transient sialadenopathy during induction of anesthesia. Anesthesiology, 29, 1050.

BLACKFORD, R.W. (1944) Recurrent swelling of the paroifd and submaxillary glands following bronchoscopy. Anng of Otology, Rhinology and Laryngology, 53, 54.

BoNCHEK, L.I. (1969) Salivary gland enlargement during induction of anesthesia. Journal of the American Medici Association, 209, 1716. 
Matsuki, A., Wakayama, S. \& Oyama, T. (1975) Acute transient swelling of the salivary glands during and following endotracheal anaesthesia. Anaesthetist, 24, 125.

Reilly, D.J. (1970) Benign transient swelling of the parotid glands following general anesthesia: 'anesthesia mumps'. Anesthesia and Analgesia, 49, 560.

ShieldS, H.M., Soloway, R.D., Long, W.B. \& Weiss, J.B.
(1977) Bilateral recurrent parotid gland swelling after endoscopy. Gastroenterology, 73, 164.

Slaughter. R.L. \& Boyce, H.W. (1969) Submaxillary salivary gland swelling developing during peroral endoscopy. Gastroenterology, 57, 83.

Slaughter, R.L. (1975) Parotid gland swelling developing during peroral endoscopy. Gastrointestinal Endoscopy, 22, 38. 